\title{
The effects of overtraining in the Morris water maze on allocentric and egocentric learning strategies in rats
}

\author{
John Kealy, Mairead Diviney, Elizabeth Kehoe, Vanessa McGonagle, Adrienne O’Shea, \\ Deirdre Harvey, Sean Commins* \\ Department of Psychology, National University of Ireland, Maynooth, Co. Kildare, Ireland
}

\section{A R T I C L E I N F O}

\section{Article history:}

Received 3 March 2008

Received in revised form 11 April 2008

Accepted 14 April 2008

Available online 20 April 2008

\section{Keywords:}

Egocentric

Allocentric

Spatial navigation

Water maze

\begin{abstract}
A B S T R A C T
Animals can use both allocentric and egocentric strategies to learn a spatial task. Our results suggest that allocentric cues are more dominant than idiothetic cues in guiding navigation. Animals do not necessarily learn an egocentric strategy automatically, instead they probably hold just one solution to any particular task at a time until forced to learn an alternative strategy. Further, with overtraining animals do not always switch from allocentric to an egocentric learning strategy perhaps challenging suggestions of a stored hierarchy of strategies.
\end{abstract}

(c) 2008 Elsevier B.V. All rights reserved.
Spatial learning and memory, vital for animals when navigating both novel and familiar environments, has been shown to rely on both egocentric $[8,13]$ and allocentric learning strategies $[9,14]$. One common test of spatial memory is the Morris water maze (MWM) $[9,10]$. The use of external allocentric cues in solving the MWM has been well documented $[4,9]$ with many researchers concluding that it seems to be the primary learning strategy employed by animals. The role of internal egocentric (or idiothetic) cues such as selfgenerated movements in spatial navigation however is less clear. Animals can learn to locate the escape platform without access to any extramaze cues [9] by developing an egocentric strategy based on self-movement information alone. This is usually achieved experimentally by allowing animals to navigate in the dark and by keeping the spatial relationship between the animal's starting position and the goal position constant (e.g. $[3,8,12])$. Although animals take longer to acquire the task using this method [10], through repeated training a procedural strategy emerges [15] and animals learn to head directly to the platform.

In solving the MWM it is proposed that rats typically use a combination of allocentric and egocentric strategies [7,8]. Furthermore, it has been suggested that rats typically engage in allocentric strategies when initially learning a location, then switch to egocentric strategies after overtraining in a task [1,11]. For example, Chang and Gold's study [1] demonstrated that as rats were trained in a cross-

\footnotetext{
* Corresponding author. Tel.: +353 1 7086182; fax: +3531 1084767

E-mail address: Sean.Commins@nuim.ie (S.Commins).
}

maze there was a switching between allocentric and egocentric strategies along with a corresponding change in neurotransmitter release in the hippocampus and striatum. In both humans and rodents allocentric memory is thought to depend on the hippocampus $[1,5]$ and egocentric memory seems to be dependent on the caudate nucleus of the striatum $[1,2,5,11]$. Thus it is postulated that animals rely initially on a hippocampally based declarative memory strategy, and then switch to a mechanism that relies on a more striatum-based procedural memory, which is incrementally learned with repeated exposure to the same task. Others however suggest a hierarchical system of strategies where rats will use visual cues over olfactory cues and olfactory cues over idiothetic cues [4]. Indeed, recent studies from our laboratory showed that when allocentric and idiothetic cues are in conflict, rats would preferentially use the allocentric cues [6,7]. However, it may not simply be a case of using allothetic cues over idiothetic cues when both are available. There is evidence that both allocentric and egocentric strategies can be utilised at the same time to different extents [3,7,11]

We previously hypothesised that if multiple forms of spatial information are used to acquire the MWM, then multiple forms of spatial information might be retained in long-term memory [6]. However, we recently demonstrated [6] that animals trained from a fixed starting position, to locate a goal in a fixed position did not seem to acquire an egocentric representation of the task. Rotation of their starting position during the probe trial did not disrupt their swimming behaviour, and after a short initial period of uncertainty (lasting just a few seconds) the animals simply used the available distal cues to search for the platform (an allocentric 
strategy). Provided that both allocentric and egocentric strategies are initially acquired, we suggested that in order for an egocentric solution alone to emerge, two changes might be made to the experimental protocol. First, the training period might be increased, and second, the visual allocentric cues might be removed from the arena for the probe trial. Here we investigate these possibilities in three experiments.

Male Wistar rats (250-350 g; Biomedical Facility, University College Dublin) aged approximately 3 months were used in all experiments. Rats were housed three/cage and kept in a temperature-controlled room that was maintained on a fixed 12:12 h light-dark cycle. All rats were given free access to food and drink. The MWM, a black circular fibreglass pool ( $1.7 \mathrm{~m}$ diameter; $38 \mathrm{~cm}$ deep), was filled with water to approximately $31 \mathrm{~cm}$ (temperature of $\left.20 \pm 1^{\circ} \mathrm{C}\right)$. Rats escaped by locating a hidden platform $(9 \mathrm{~cm}$ diameter, $29 \mathrm{~cm}$ height) located in the middle of northeast quadrant. The platform was submerged $2 \mathrm{~cm}$ below the water surface, rendering it invisible to the rats when swimming. The pool was surrounded by a black curtain which was located approximately $50 \mathrm{~cm}$ from the pool wall. Distal cues included two $60 \mathrm{~W}$ lights suspended from the ceiling, hanging within the surrounding curtain (located in the northwest and northeast corners, respectively). A rectangular sheet of white paper $(55 \mathrm{~cm} \times 81 \mathrm{~cm})$ was also attached to the curtain on the east side of the pool for use as a cue. All movements and escape latencies were recorded by using EthoVision (Noldus, Wageningen, Netherlands).

In experiment 1 , rats $(n=30)$ were trained with access to distal cues to find the hidden platform (NE) from a fixed starting position (NW) over 4 days ( 4 trials/day). Seven days post-acquisition animals were randomly divided into four groups, retention was then tested by a probe trial by allowing animals to search in a single $60 \mathrm{~s}$ trial in a platform-less pool. Mean percentage time (of $60 \mathrm{~s}$ ) in various regions of the maze was used to assess retention (see [7] for details). Groups 1 and 2 were re-tested in the light with cues present but Group 1 (control/light, $n=7$ ) was placed into the pool from the trained starting position (i.e. NW) and Group 2 began the probe trial from a position $180^{\circ}$ away from this (start-rotated (S$\mathrm{R}$ )/light, $n=8$ ). Groups 3 and 4 consisted of a control and S-R group but both of these groups were retested in the dark with cues absent ( $n=7$ and 8, respectively). We hypothesised that if an egocentric strategy was learned in conjunction with an allocentric one then both S-R groups would search less in NE quadrant than the nonrotated groups and that the S-R/dark group would rely completely on a procedural strategy and search in the opposite quadrant (SW). In experiment 2 we used the same four conditions for retention ( $n=7,8,8$ and 8 for Groups $1-4$, respectively) but animals were trained for 12 days instead of 4 . If a procedural strategy did not emerge following 4 days of training we would expect it to emerge with a longer training regime. Whereas in experiments 1 and 2 the retention of some groups was conducted in the dark, in experiment 3 to ensure that the animals could still recognise the testing environment and allowed to search the pool without access to the previously learned distal cues, we conducted the retention trial in a bright cueless environment. Animals were trained for 12 days ( 4 trials/day). Seven days post-acquisition, animals were re-tested. For the retention trial all distal cues were completely removed and were subsequently replaced by a single $60 \mathrm{~W}$ light bulb suspended from the ceiling directly above the centre of the pool. Immediately prior to the retention trial animals were randomly assigned into one of two groups. The control group $(n=8)$ was placed into the water maze from the NW quadrant, as they were during the acquisition phase. The S-R group $(n=8)$ was placed into the water maze from the $\mathrm{SE}$ quadrant, a $180^{\circ}$ rotation compared to their starting point in the acquisition phase. Retention analysis was conducted similar to the previous experiments. All statistics were carried out using SPSS
(V.10). All experiments were carried out in accordance with the European Communities Council Directive (86/609/EEC) and with Irish Department of Health and Children regulations.

Training over 4 days in experiment 1 led to all animals acquiring the water maze task (Fig. $1 \mathrm{a}, F=42.959$; d.f. $=3,87 ; p<0.001$ ). Fig. 1b demonstrates that both the control and S-R groups tested in the light searched the NE area significantly more than the control and S-R groups tested in the dark. A $2 \times 2$ between groups ANOVA found no overall significant effect for the control vs. S-R condition $(F=0.002$; d.f. $=1,26 ; p>0.05)$. There was however, an effect of light vs. dark $(F=24.655$; d.f. $=1,26 ; p<0.001)$. In addition, no interaction effect $(F=0.012$; d.f. $=1,26 ; p>0.05)$ was found. Independent $t$-tests found that the control/light group searched for the platform in the NE area significantly more than the control/dark group $(t=3.85$; d.f. $=12 ; p<0.01)$, with the $\mathrm{S}-\mathrm{R} /$ light group also exploring this area significantly more than the $S-R /$ dark group (Fig. 1b, $t=3.308$; d.f. $=14 ; p<0.01$ ). Comparing the control/light and $\mathrm{S}-\mathrm{R} /$ light groups, no significant differences were found between the groups with respect to their time exploring either the NE area $(t=0.40$; d.f. $=13 ; p>0.05)$ or the full NE quadrant $(t=0.898$; d.f. $=13 ; p>0.05)$ or any other subregion within the NE quadrant (data not shown). This would suggest that rotating the starting position $180^{\circ}$ did not impair the animal's ability to search for the platform in the NE region, suggesting that the S-R (light) group simply used the available distal cues. We had hypothesised that if a procedural strategy was acquired and retained in conjunction with an allocentric strategy during learning, then, in the dark conditions animals would be required to adopt a procedural strategy, i.e. the control group would search in the NE quadrant/area and the S-R group in the SW quadrant/area. We found however this is not to be the case. Animals in both dark conditions spent significantly more time at the side of the pool, remaining particularly at their own respective starting positions compared to the light groups (Fig. 1c and d). We compared each group on the mean percentage time spent swimming in the outer corridor (Fig. 1c). A significant effect was found for both lighting condition (light vs. dark, $F=21.542$; d.f. $=1,26 ; p<0.001$ ) and group type (control vs. S-R, $F=6.175$; d.f. $=1,26 ; p<0.05)$ but no interaction effects were noted $(F=2.240$; d.f. $=1,26 ; p>0.05)$. We also compared the four groups on the time spent in their respective starting positions (see Fig. 1d). Again, an overall significant effect for lighting condition was found $(F=5.094$; d.f. $=1,26 ; p<0.05)$, demonstrating that both dark groups spent more time where they started from compared to the light. There was neither an overall effect for group (control vs. S$\mathrm{R}, F=0.439$; d.f. $=1,26 ; p>0.05)$ nor an interaction effect $(F=2.551$; d.f. $=1,26 ; p>0.05)$ suggesting that animals in the S-R conditions explored similarly to the control groups.

In experiment 2 training over 12 days led to all animals successfully acquiring the water maze task. An overall significant decrease in escape latency $(F=81.188$; d.f. $=11,330 ; p<0.001)$ across the 12 days (Fig. 2a) was found. Similar to the pattern observed in searching behaviour following 4 days of training, Fig. 2 b demonstrates that both light groups searched significantly more than the two dark groups in the NE area with a significant main effect for lighting conditions $(F=49.956$; d.f. $=1,27 ; p<0.001)$ but neither a group nor an interaction effect $(F=1.175$; d.f. $=1,27 ; p>0.05$ and $F=0.693$; d.f. $=1$, $27 ; p>0.05$, respectively). We found that the control/light group searched significantly more than the control/dark group in the NE area $(t=5.465$; d.f. $=13 ; p<0.001)$, similarly the $\mathrm{S}-\mathrm{R} /$ light group also searched significantly more $(t=4.508$; d.f. $=14 ; p<0.001)$ in the NE area compared to the $\mathrm{S}-\mathrm{R} /$ dark group. Interestingly, no significant differences were noted between the control/light or S-R/light group in the amount of time spent searching for the platform in either the NE area $(t=1.049$; d.f. $=13 ; p>0.05)$, the full NE quadrant $(t=0.848$; d.f. $=13 ; p>0.05$ ), or any other region of the NE quadrant (data not 

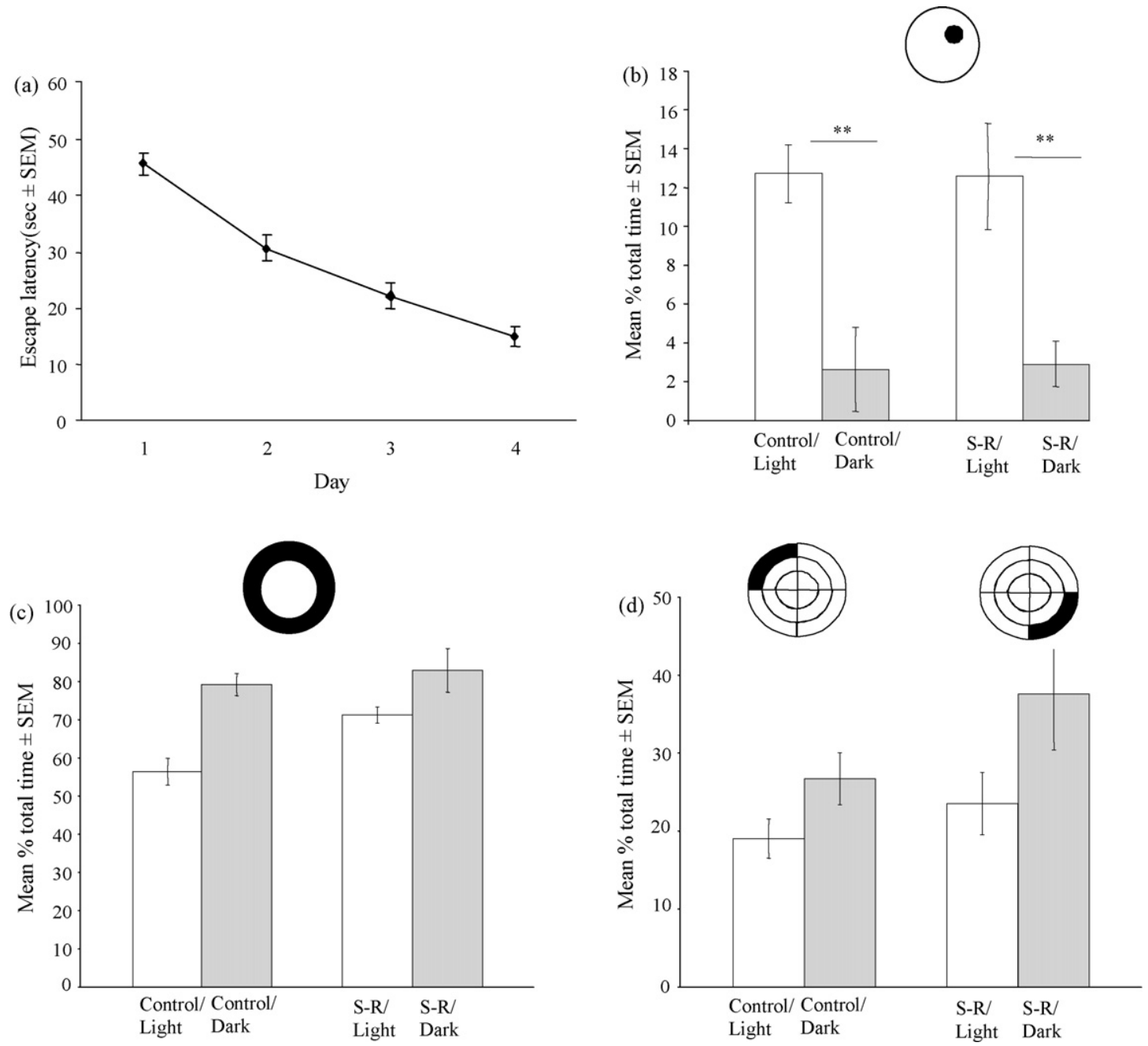

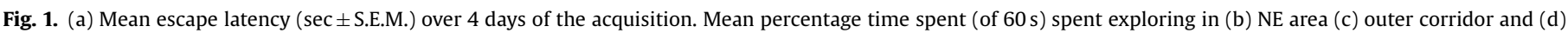
starting position for all groups during the retention phase of the water maze task. ${ }^{* *} p<0.01$.

shown) suggesting that despite extra training, the S-R group did not swim to the SW quadrant to look for the platform.

In addition, similar to the results reported in experiment 1 animals exposed to the retention trial in the dark displayed thigmotaxis-like behaviour and swam around the side of the pool despite having extra training. Fig. 2c demonstrates this where both dark groups spent approximately $80 \%$ of the time in the outer corridor. We found a significant effect for lighting conditions $(F=58.107$; d.f. $=1,27 ; p<0.001)$ but no effect for group $(F=0.091$; d.f. $=1,27 ; p>0.05)$ or interaction $(F=0.973$; d.f. $=1,27 ; p>0.05)$. Two independent $t$-tests confirmed that the control/dark group spent significantly greater time in the outer corridor compared with the control/light group $(t=-4.960$; d.f. $=13 ; p<0.001)$, similarly, the $\mathrm{S}-\mathrm{R} /$ dark group also spent significantly more time in this region compared to the $\mathrm{S}-\mathrm{R} /$ light group $(t=-5.841$; d.f. $=14$; $p<0.001)$. An overall significant effect for lighting condition was found $(F=23.378$; d.f. $=1,27 ; p<0.001)$ when we compared the groups on the time spent in their respective starting positions (Fig. 2d). There was also an overall effect for group (control vs. S-R: $F=5.845$; d.f. $=1,27 ; p<0.05)$ but no interaction effect $(F=0.934$; d.f. $=1,27 ; p>0.05$ ).

All animals successfully acquired the water maze task in experiment 3 with an overall significant decrease in escape latency $(F=42.257$; d.f. $=11,165 ; p<0.001)$ across the 12 days (Fig. 3a).
Fig. 3b demonstrates that both groups spent equivalent time swimming in all four quadrants. A small quadrant effect $(F=3.824$; d.f. $=3$, $56 ; p<0.05)$ but no group $(F=0.001$; d.f. $=1,56 ; p>0.05)$ or interaction effect $(F=1.028$; d.f. $=3,56 ; p>0.05)$ was found. Post hoc (Tukey, $p<0.05)$ tests suggest that the groups swam significantly more in the SE quadrant compared to the SW. No other differences were noted. Furthermore, the control group neither show a preference for the NE quadrant nor the S-R group a preference for the SW quadrant as would be expected if they adopted a procedural strategy. Indeed, the S-R group did not swim for the NE quadrant, a strategy that we had previously observed for the light groups in experiments 1 and 2 . Rather, both the control and the S-R groups had a tendency to swim around the side of the pool, but in a pattern different to the one adopted by the groups completely in experiment 2 . Animals in the dark swam at the side of the pool in a thigmotactic fashion, whereas animals in a bright cue-less environment swam both at the side of the pool and also around an inner corridor of the pool, at a distance equivalent to the location of the platform relative to the pool side. Both control and S-R dark groups (of experiment 2) spent significantly more time swimming in the outer corridor (at side of pool) compared to the cue-less groups ( $F=22.239$; d.f. $=1,28$; $p<0.001)$, whereas both cue-less groups spent significantly more time in an inner corridor of the pool compared to the dark groups $(F=18.153 ;$ d.f. $=1,28 ; p<0.001)$. 

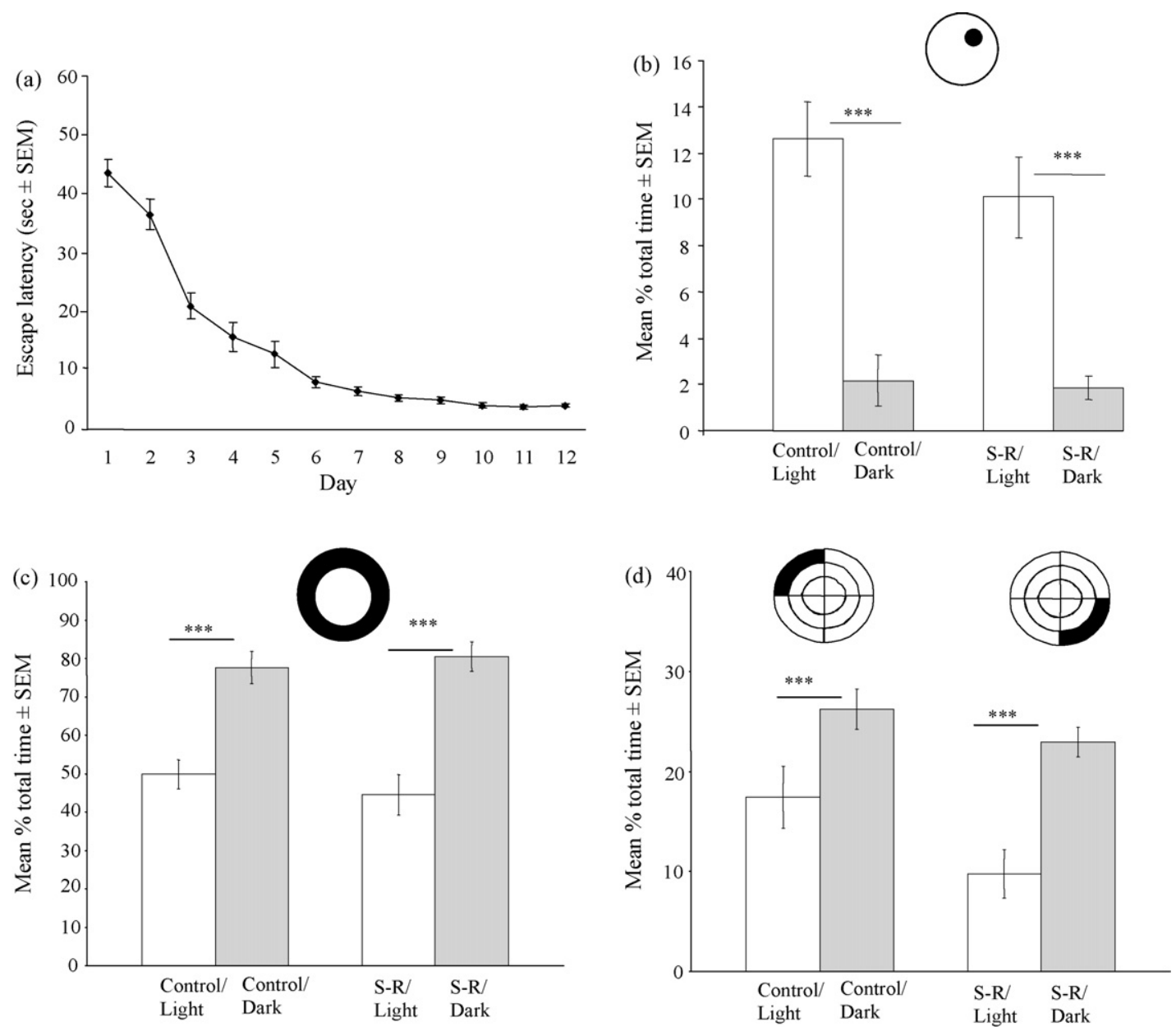

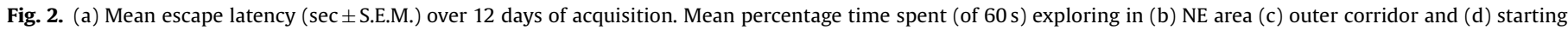
position for all groups during the retention phase of the water maze task. $p<0.01,{ }^{* * *} p<0.001$.

Our experiments suggest that animals in the MWM rely heavily on the distal visual cues to locate the submerged platform, this is despite being trained to a fixed goal from a constant start position. Animals placed into the pool at a position $180^{\circ}$ to which they had been trained, followed the cues and searched in the NE quadrant rather than in the SW quadrant as would have been appropriate if they had acquired a procedural strategy. This result might be expected as we have previously demonstrated that if a conflict arises between allocentric and egocentric strategies, animals would tend to rely on an allocentric strategy and follow the cues [6]. However, if animals had acquired an egocentric strategy in conjunction with the allocentric one, then animals placed into the pool in the dark would be forced to adopt the egocentric one, either swimming to the NE quadrant for the control group or the SW for the S-R group, but this did not prove to be the case. Animals, irrespective of what group that they been assigned to, either simply swam around the side of the pool or stayed at their respective starting position.

We also found that overtraining on the task (12 days) also did not lead to the acquisition of an egocentric, procedural solution, rather results from this experiment suggest that animals reliance on the cues increased as opposed to developing or switching to a more procedural strategy. The only difference that we observed between animals trained for 12 days as opposed to just 4 days was that the animals trained for 12 days spent a longer period of time in the NE region. In addition, animals in the S-R condition spent less time at the side of the pool generally and less time at their starting position. Furthermore, animals in the dark groups displayed a similar pattern of thigmotactic-like behaviour as was observed with just 4 days of training. In experiment 3 we removed the possibility that animals may simply did not recognise the training environment when required to swim in the dark. So the probe task was conducted in a bright cueless environment. However, we found that animals despite being trained for 12 days did not adopt a procedural strategy. Rather, the findings from this experiment reinforce the idea of the reliance of the distal cues to accurately locate the hidden platform. In addition, animals seem to use the pool wall as a cue, swimming randomly at the correct distance from the side.

Many authors would suggest that rats typically use a combination of allocentric and egocentric strategies to solve the MWM [7,8]; employing both distal cues and self-movement feedback; however the relative contributions of these mechanisms is not well understood [3]. Furthermore, it has been suggested that rats typically engage in allocentric strategies when learning locations, then switch to egocentric strategies after overtraining [1,11]. We however found that overtraining did not lead to the adoption of a procedural-based strategy despite been trained from a fixed starting position to a fixed goal for 12 days. It is possible that such an egocentric strategy can only emerge during testing if it had been learned during training. As the visual cues were available to animals for the entire training period, perhaps animals became over reliant on these cues and therefore a fixed motor plan was never acquired, thus animals became totally disorientated when the cues were no longer available. It is also possible the water maze is not ideal task to train a motor plan; this task allows too much flexi- 

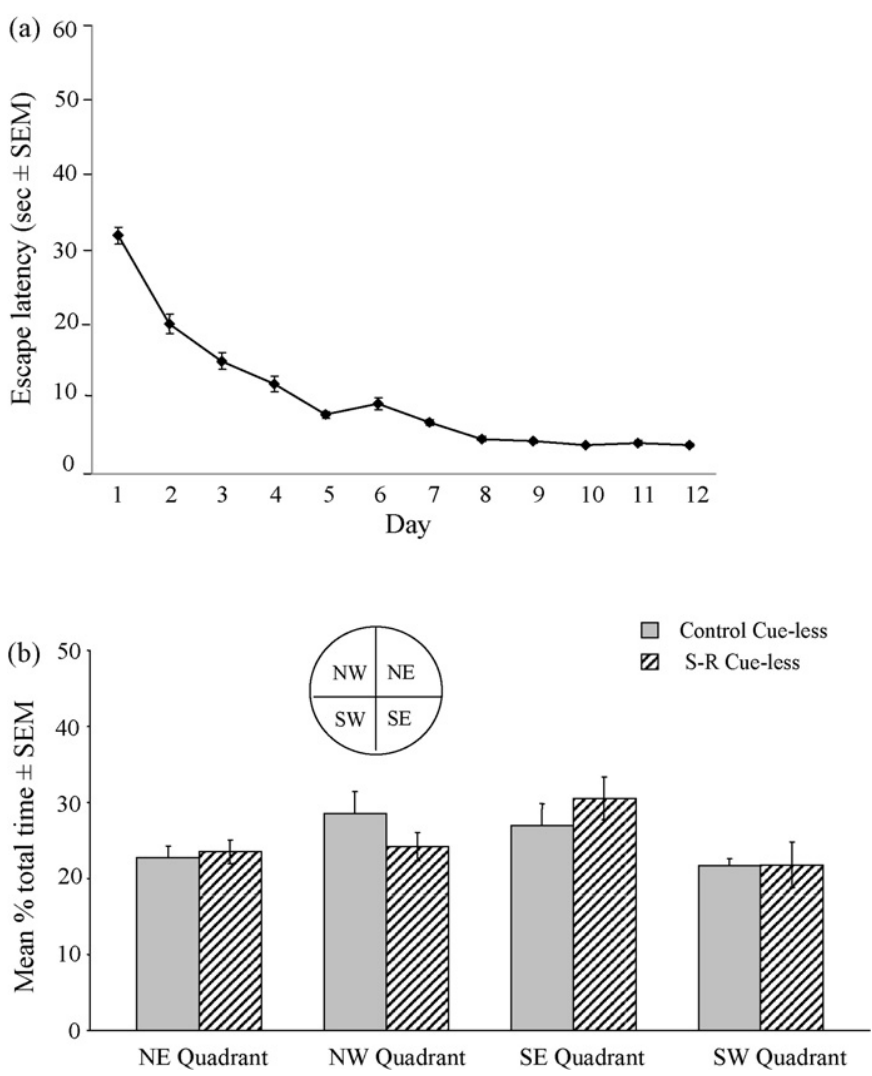

Fig. 3. (a) Mean escape latency ( $\mathrm{sec} \pm$ S.E.M.) over 12 days of acquisition. (b) Bar chart demonstrating the mean percentage time spent by the control cue-less group (grey bars) and the S-R cue-less group (hatched bars) in each of the four quadrants of the pool.

bility in movement. Procedural learning may emerge more rapidly where there is a constraint on the animal's motor movement, e.g. in a cross-maze [1].

Our results also question whether an animal necessarily holds more than one solution to a given navigational task, and that on occasion of one strategy becoming ineffective due to changed environmental conditions, a back-up system should be flexibly available to guide navigation. In our experiments it was predicted that the removal of distal visual cues, presumably forming the basis of an allocentric spatial solution might allow an egocentric strategy based on self-movement cues to emerge, this did not seem to occur. Although the storage of back-up solutions of a task might be advisable in case of the failure of one system, from the perspective of the animal's cognitive system, it might not make economical sense to store multiple representations of a task when only one is needed.
Maaswinkel and Whishaw [4] suggest that a hierarchy of strategies are available to an animal. In addition, they reported that the animals could flexibly combine, and switch between using cues when another set was not available. Our results would suggest that this might not necessarily be true, at least in the water maze experiments. However, as outlined above, in the case of our study, no poverty of availability of visual cues was experienced until the testing phase, and so the animals up until this point had no need for a back-up egocentric navigational system, when the visually based, allocentric one was sufficient for locating the platform in every trial.

\section{Acknowledgements}

This work was supported by Science Foundation of Ireland (SFI) and the Irish Research Council for Science, Engineering and Technology (IRCSET). The authors would also like to thank Dr. AnneMarie McGauran (NUIM) for her contributions.

\section{References}

[1] Chang Q Gold PE. Switching memory systems during learning: changes in patterns of brain acetylcholine release in the hippocampus and striatum in rats. J Neurosci 2003;23:3001-5.

[2] Cook D, Kesner RP. Caudate nucleus and memory for egocentric localization. Behav Neural Biol 1988;49:332-43.

[3] Gerlai RT, McNamara A, Williams S, Phillips HS. Hippocampal dysfunction and behavioral deficit in the water maze in mice: an unresolved issue? Brain Res Bull 2002;57:3-9.

[4] Maaswinkel H, Whishaw IQ. Homing with locale, taxon, and dead reckoning strategies by foraging rats: sensory hierarchy in spatial navigation. Behav Brain Res 1999;99:143-52.

[5] McDonald RJ, White NM. Parallel information processing in the water maze: evidence for independent memory systems involving dorsal striatum and hippocampus. Behav Neural Biol 1994;61:260-70.

[6] McGauran AT, Harvey D, Cunningham D, Craig S, Commins S. Retention of cue-based associations in the water maze is time-dependent and sensitive to disruption by rotating the starting position. Behav Brain Res 2004;151:255-66.

[7] McGauran AT, O’Mara SM, Commins S. Vestibular influence on water maze retention: transient whole body rotations improve the accuracy of the cuebased retention strategy. Behav Brain Res 2005;158:183-7.

[8] Moghaddam M, Bures J. Contribution of egocentric spatial memory to place navigation of rats in the Morris water maze. Behav Brain Res 1996;78:121-9.

[9] Morris RGM. Spatial localization does not require the presence of local cues. Learn Motiv 1981;12:239-60.

[10] Morris RGM. Development of a watermaze procedure for studying spatial learning in the rat. J Neurosci Methods 1984;11:47-60.

[11] Packard MG, McGaugh JL. Inactivation of hippocampus or caudate nucleus with lidocaine differentially affects expression of place and response learning. Neurobiol Learn Memory 1996;65:65-72.

[12] Wallace DG, Hines DJ, Pellis SM, Whishaw IQ. Vestibular information is required for dead reckoning in the rat. J Neurosci 2002;22(22):10009-17.

[13] Wang RF, Spelke ES. Human spatial representation: insights from animals. Trends Cogn Sci 2002;6:376-82.

[14] Warburton EC, Baird AL, Aggleton JP. Assessing the magnitude of the allocentric spatial deficit associated with complete loss of the anterior thalamic nuclei in rats. Behav Brain Res 1997;87:223-32.

[15] Whishaw IQ. Cholinergic receptor blockade in the rat impairs locale but not taxon strategies for place navigation in a swimming pool. Behav Neurosci 1985;99(5):979-1005. 